\title{
EXISTENCE AND UNIQUENESS FOR DYNAMICAL UNILATERAL CONTACT WITH COULOMB FRICTION: A MODEL PROBLEM
}

\author{
PATRICK BAllard $^{1}$ AND StéPhanie Basseville ${ }^{1}$
}

\begin{abstract}
A simple dynamical problem involving unilateral contact and dry friction of Coulomb type is considered as an archetype. We are concerned with the existence and uniqueness of solutions of the system with Cauchy data. In the frictionless case, it is known [Schatzman, Nonlinear Anal. Theory, Methods Appl. 2 (1978) 355-373] that pathologies of non-uniqueness can exist, even if all the data are of class $C^{\infty}$. However, uniqueness is recovered provided that the data are analytic [Ballard, Arch. Rational Mech. Anal. 154 (2000) 199-274]. Under this analyticity hypothesis, we prove the existence and uniqueness of solutions for the dynamical problem with unilateral contact and Coulomb friction, extending [Ballard, Arch. Rational Mech. Anal. 154 (2000) 199-274] to the case where Coulomb friction is added to unilateral contact.
\end{abstract}

Mathematics Subject Classification. 34A60, 49J52, 70F40.

Received: May 24, 2004. Revised: November 4, 2004.

\section{DESCRIPTION OF THE PROBLEM}

At the time being, dynamics involving unilateral contact and Coulomb friction has been mainly studied in the framework of systems with finite number of degrees of freedom. In this paper, we are concerned with the questions of existence and uniqueness of solutions for the associated evolution problem. In order to make clear the structure of our existence and uniqueness proof, we shall consider only the simple system introduced by Klarbring [4]. However, the reader should have in mind that the proof presented here can be adapted to a far more general situation. The most general situation in finite d.o.f. dynamics with unilateral contact and Coulomb friction, which is covered by our approach, will be described in a next publication.

Klarbring's system refers to the following situation. Let $n \geq 2$ be some integer. A punctual particle of unit mass in $\mathbb{R}^{n}$ evolves in a quadratic well of potential elastic energy, described by a symmetric positive definite stiffness matrix $K$, and is subjected to an external force $F(t)$, depending only on time. Moreover, the particle is constrained to remain in a half-space, and, at contact, Coulomb friction takes place. For $X \in \mathbb{R}^{n}$, we denote by $X_{\mathrm{N}}$ its first component ("normal component") and by $X_{\mathrm{T}}$ the vector of $\mathbb{R}^{n-1}$ formed by the $n-1$ last components of $X$ ("tangential component"). The symmetric positive definite stiffness matrix $K$ will be written as:

$$
K=\left(\begin{array}{cc}
k_{\mathrm{N}} & { }^{t} W \\
W & K_{\mathrm{T}}
\end{array}\right)
$$

Keywords and phrases. Unilateral dynamics with friction, existence and uniqueness.

1 Laboratoire de Mécanique et d'Acoustique, 31, Chemin Joseph Aiguier, 13402 Marseille Cedex 20, France.

ballard@lma.cnrs-mrs.fr

(C) EDP Sciences, SMAI 2005 


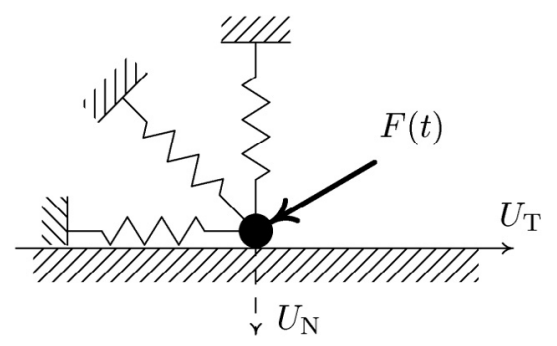

FiguRE 1. Klarbring's system.

where $k_{\mathrm{N}} \in \mathbb{R}, W \in \mathbb{R}^{n-1}$ and $K_{\mathrm{T}}$ is a symmetric positive definite real matrix of order $n-1$. The two following statements are equivalent.

(i) The matrix $K$ is positive definite.

(ii) The matrix $K_{\mathrm{T}}$ is positive definite and $k_{\mathrm{N}}>{ }^{t} W \cdot K_{\mathrm{T}}^{-1} \cdot W$.

The term $W$ couples the normal and tangential degrees of freedom and is a source of difficulty in the analysis of the system.

We denote by $\operatorname{MMA}\left([0, T] ; \mathbb{R}^{n}\right)$ (motions with measure acceleration) the vector space of those integrable functions of $[0, T]$ into $\mathbb{R}^{n}$ whose second derivative in the sense of distributions is a measure. It is nothing but the space of integrals of functions of bounded variation over $[0, T]$. Functions $U$ in $M M A$ are continuous and admit left and right derivatives (in the classical sense) $\dot{U}^{-}, \dot{U}^{+}$, at any point, both being functions of bounded variation. We recall that a function of bounded variation, being uniform limit of a sequence of step functions, is universally integrable (integrable with respect to any measure). The evolution problem is formulated along the lines of Moreau [6,8] and in the sequel, the term "unilateral problem" will refer to the following evolution problem:

Problem $\mathcal{P}_{\mathbf{u}}$. Find $U \in M M A\left([0, T] ; \mathbb{R}^{n}\right)$ and $R \in \mathcal{M}\left([0, T] ; \mathbb{R}^{n}\right)$ such that:

- $U(0)=U_{0} ; \quad \dot{U}^{+}(0)=V_{0} \quad$ (initial condition);

- $\ddot{U}+K \cdot U=F+R, \quad$ in $[0, T] \quad$ (motion equation);

- $U_{\mathrm{N}} \leq 0, \quad R_{\mathrm{N}} \leq 0, \quad U_{\mathrm{N}} R_{\mathrm{N}}=0 \quad$ (unilateral contact);

- $\int_{[0, T]}\left[R_{\mathrm{T}} \cdot\left(V-\dot{U}_{\mathrm{T}}^{+}\right)-\mu R_{\mathrm{N}}\left(|V|-\left|\dot{U}_{\mathrm{T}}^{+}\right|\right)\right] \geq 0, \quad \forall V \in C^{0}\left([0, T] ; \mathbb{R}^{n-1}\right) \quad$ (Coulomb friction);

- $U_{\mathrm{N}}(t)=0 \quad \Longrightarrow \quad \dot{U}_{\mathrm{N}}^{+}(t)=-e \dot{U}_{\mathrm{N}}^{-}(t), \quad$ in $\left.] 0, T\right] \quad$ (impact law),

where $F$ denotes a given integrable function of $[0, T]$ into $\mathbb{R}^{n}$ (external force), $\mu$ a given nonnegative real constant (friction coefficient), $e \in[0,1]$ a real constant (restitution coefficient) and $\left(U_{0}, V_{0}\right)$ some initial condition, assumed to be compatible with the unilateral constraint:

$$
U_{0 \mathrm{~N}} \leq 0 \quad \text { and: } \quad U_{0 \mathrm{~N}}=0 \quad \Longrightarrow \quad V_{0 \mathrm{~N}} \leq 0
$$

Our goal is to investigate the existence and uniqueness of a solution of problem $\mathcal{P}_{\mathbf{u}}$.

\section{REVIEW OF EXISTING RESUlTS AND CONTENT}

Well-posedness of the dynamics of discrete systems with unilateral constraints (without friction) seems to have been first investigated by Schatzman in [11], where she proved an existence result by a penalization 
technique in the case of the elastic impact law $e=1$. She also gave a striking counter-example showing that, even in the case where the data have regularity $C^{\infty}$, one cannot expect uniqueness of solution, in general. A major remark was, then, made by Percivale in [10] who noticed that, in the case of the (necessarily frictionless) one-degree-of-freedom problem with external force depending only on time, uniqueness of solution is recovered provided the external force is assumed to be an analytic function of time (instead of $C^{\infty}$ ). Later, Schatzman [12] provided a generalization of this uniqueness result under analyticity assumption, still for the one-degreeof-freedom problem, but in the more general case where the external force is allowed to depend not only on time but also on current position and velocity. However, her proof was specific to the one-degree-of-freedom problem. A simpler proof was given by Ballard [1] who was, then, able to extend the result to the general case of an arbitrary number of degrees of freedom and unilateral constraints. But his result was restricted to the frictionless situation.

The case of dry friction has been considered by Monteiro Marques in [5]. He considered a case with a single smooth unilateral constraint and inelastic impact law $e=0$ which contains Klarbring's system (provided $e=0)$. Using a time-stepping algorithm introduced by Moreau [7,8] (which is, roughly speaking, an adaptation of the implicit Euler scheme to the non-smooth situation under consideration) to build a sequence of approximants, Monteiro Marques was able to pass to the limit by extraction of a subsequence using a compactness argument, to prove an existence result which applies to Klarbring's system in the case $e=0$ and $F \in L^{1}$. However, note that Klarbring's system, in the particular case $W=0$ and $F_{\mathrm{T}} \equiv 0$, reduces to a one-degree-of freedom system in which Coulomb friction plays no role, and, Schatzman's counter-example [11] can be readily adapted to this particular case of Klarbring's system, demonstrating that one cannot expect uniqueness in general, even in the case where the external force is assumed to have $C^{\infty}$ regularity . Therefore, our purpose, here, is to adapt the technique of Ballard [1] to the situation where Coulomb friction is involved, to prove the uniqueness of a solution under the assumption that $F$ is an analytic function of time.

In the frictionless situation, Ballard's uniqueness proof relied on the fact that the associated bilateral problem is governed by an ordinary differential equation, whose solution is analytic provided the data of the problem are analytic. In the situation under consideration, the associated bilateral problem is governed by a differential inclusion (multivocal differential equation) because of Coulomb friction. The Cauchy problem associated with the bilateral problem is studied in Section 3. First, the existence and uniqueness of a solution is proved by use of standard monotonicity techniques in Section 3.1. Then, it is proved in Section 3.2 that the restriction of the solution on some right-neighbourhood of the time origin is analytic, provided the external force is analytic. The analysis of the bilateral problem, as performed in Section 3, is used in Section 4.1 to build a local analytic solution of the unilateral problem with analytic external force. Then, to obtain well-posedness for the unilateral problem, there remains only to prove that there cannot exist any other local solution in $M M A$, different from the local analytic one. This is performed in Section 4.2 by adapting Ballard's strategy [1] to the situation under consideration.

\section{The Bilateral problem}

In the sequel, the "bilateral problem" will refer to the evolution problem we obtain when the unilateral constraint is replaced by a bilateral constraint. More precisely, this is the following evolution problem.

Problem $\mathcal{P}_{\mathbf{b}}$. Find $U \in M M A\left([0, T] ; \mathbb{R}^{n}\right)$ and $R \in \mathcal{M}\left([0, T] ; \mathbb{R}^{n}\right)$ such that:

- $U(0)=U_{0} ; \quad \dot{U}^{+}(0)=V_{0} \quad$ (initial condition);

- $\ddot{U}+K \cdot U=F+R, \quad$ in $[0, T] \quad$ (motion equation);

- $U_{\mathrm{N}} \equiv 0 \quad$ (bilateral contact);

- $\int_{[0, T]}\left[R_{\mathrm{T}} \cdot\left(V-\dot{U}_{\mathrm{T}}^{+}\right)+\mu\left|R_{\mathrm{N}}\right|\left(|V|-\left|\dot{U}_{\mathrm{T}}^{+}\right|\right)\right] \geq 0, \quad \forall V \in C^{0}\left([0, T] ; \mathbb{R}^{n-1}\right) \quad$ (Coulomb friction), 
where $F$ denotes some given integrable function $\left(F \in L^{1}([0, T] ; \mathbb{R})\right)$ and $\left(U_{0}, V_{0}\right)$ some initial condition, assumed to be compatible with the bilateral constraint:

$$
U_{0 \mathrm{~N}}=0 \quad \text { and } \quad V_{0 \mathrm{~N}}=0 .
$$

Actually, the first component of the motion equation:

$$
R_{\mathrm{N}}=W \cdot U_{\mathrm{T}}-F_{\mathrm{N}},
$$

shows that the measure $R_{\mathrm{N}}$ is necessarily absolutely continuous with respect to the Lebesgue measure. Since the Coulomb friction law implies the following inequality between measures:

$$
\left|R_{\mathrm{T}}\right| \leq \mu\left|R_{\mathrm{N}}\right|
$$

we infer that the measure $R_{\mathrm{T}}$ is also absolutely continuous with respect to the Lebesgue measure. As a result, any solution $(U, R) \in M M A \times \mathcal{M}$ of problem $\mathcal{P}_{\mathrm{b}}$ belongs, actually, to $W^{2,1} \times L^{1}$. For $\mathcal{C}$ being a nonempty closed convex subset of $\mathbb{R}^{n-1}$, we denote by $\partial S_{\mathcal{C}}$ the subdifferential of its support function $S_{\mathcal{C}}$. In the sequel, $\mathcal{B}$ will be the closed unit ball of Euclidean $\mathbb{R}^{n-1}$. Using these notations, we have the following equivalent formulation for problem $\mathcal{P}_{\mathrm{b}}$.

Problem $\mathcal{P}_{\mathbf{b}}$. Find $U_{\mathrm{T}} \in W^{2,1}\left([0, T] ; \mathbb{R}^{n-1}\right)$ such that:

- $U_{\mathrm{T}}(0)=U_{0 \mathrm{~T}} ; \quad \dot{U}_{\mathrm{T}}(0)=V_{0 \mathrm{~T}} \quad$ (initial condition);

- $\ddot{U}_{\mathrm{T}}(t)+K_{\mathrm{T}} \cdot U_{\mathrm{T}}(t)-F_{\mathrm{T}}(t) \in \partial S_{\mu\left|F_{\mathrm{N}}(t)-W \cdot U_{\mathrm{T}}(t)\right| \mathcal{B}}\left[-\dot{U}_{\mathrm{T}}(t)\right], \quad$ for a.a. $t \in[0, T]$.

\subsection{The bilateral problem with integrable force}

In this section, we prove the existence and uniqueness of a solution of problem $\mathcal{P}_{\mathbf{b}}$ by monotonicity techniques.

Proposition 3.1. Let $r \in \mathbb{R}^{+}$and $F_{\mathrm{T}} \in L^{1}\left([0, T] ; \mathbb{R}^{n-1}\right)$. Then, there exists a unique $U_{\mathrm{T}} \in W^{2,1}\left([0, T] ; \mathbb{R}^{n-1}\right)$ such that:

$$
\begin{array}{ll}
\text { - } & U_{\mathrm{T}}(0)=U_{0 \mathrm{~T}} ; \quad \dot{U}_{\mathrm{T}}(0)=V_{0 \mathrm{~T}} \quad \text { (initial condition); } \\
\text { - } & \ddot{U}_{\mathrm{T}}(t)+K_{\mathrm{T}} \cdot U_{\mathrm{T}}(t)-F_{\mathrm{T}}(t) \in \partial S_{r \mathcal{B}}\left[-\dot{U}_{\mathrm{T}}(t)\right], \quad \text { for a.a. } t \in[0, T] .
\end{array}
$$

Proof.

Uniqueness. Straightforward by monotonicity of the subdifferential.

Existence. We shall use a Caratheodory type construction, implicit with respect to the subdifferential term. Let $V_{\mathrm{T}}^{n}$ be the sequence of functions in $W^{1,1}\left([0, T] ; \mathbb{R}^{n-1}\right)$ defined by:

$$
V_{\mathrm{T}}^{0} \equiv V_{0 \mathrm{~T}}
$$

and by the following induction. Given the function $V_{\mathrm{T}}^{n} \in W^{1,1}\left([0, T] ; \mathbb{R}^{n-1}\right)$, the function $V_{\mathrm{T}}^{n+1}$ is defined to be the unique solution in $W^{1,1}\left([0, T] ; \mathbb{R}^{n-1}\right)$, provided by Proposition 3.4, p. 69 of [2], of the evolution problem:

- $V_{\mathrm{T}}^{n+1}(0)=V_{0 \mathrm{~T}}$;

- $\dot{V}_{\mathrm{T}}^{n+1}(t)+K_{\mathrm{T}} \cdot\left(U_{0 \mathrm{~T}}+\int_{0}^{t} V_{\mathrm{T}}^{n}(s) \mathrm{d} s\right)-F_{\mathrm{T}}(t) \in \partial S_{r \mathcal{B}}\left[-V_{\mathrm{T}}^{n+1}(t)\right], \quad$ for a.a. $t$. 
By monotonicity of the subdifferential, it is easily seen that, for all $t \in[0, T]$ :

$$
\frac{1}{2}\left|V_{\mathrm{T}}^{n+1}(t)-V_{\mathrm{T}}^{n}(t)\right|^{2}+\int_{0}^{t}\left[V_{\mathrm{T}}^{n+1}(s)-V_{\mathrm{T}}^{n}(s)\right] \cdot K_{\mathrm{T}} \cdot \int_{0}^{s}\left[V_{\mathrm{T}}^{n}(\sigma)-V_{\mathrm{T}}^{n-1}(\sigma)\right] \leq 0
$$

where $|\cdot|$ denotes the Euclidean norm in $\mathbb{R}^{n-1}$. Using the same notation for the associated matrix norm, we get:

$$
\frac{1}{2}\left|V_{\mathrm{T}}^{n+1}(t)-V_{\mathrm{T}}^{n}(t)\right|^{2} \leq\left|K_{\mathrm{T}}\right| \int_{0}^{t}\left|V_{\mathrm{T}}^{n+1}(s)-V_{\mathrm{T}}^{n}(s)\right| \int_{0}^{s}\left|V_{\mathrm{T}}^{n}(\sigma)-V_{\mathrm{T}}^{n-1}(\sigma)\right|,
$$

and, by Lemma A.5, p. 157 of [2]:

$$
\begin{aligned}
\left|V_{\mathrm{T}}^{n+1}(t)-V_{\mathrm{T}}^{n}(t)\right| & \leq\left|K_{\mathrm{T}}\right| \int_{0}^{t} \int_{0}^{s}\left|V_{\mathrm{T}}^{n}(\sigma)-V_{\mathrm{T}}^{n-1}(\sigma)\right| \\
& \leq\left|K_{\mathrm{T}}\right| T \int_{0}^{t}\left|V_{\mathrm{T}}^{n}(s)-V_{\mathrm{T}}^{n-1}(s)\right| \\
& \leq \frac{\left(\left|K_{\mathrm{T}}\right| T t\right)^{n}}{n !}\left\|V_{\mathrm{T}}^{1}-V_{\mathrm{T}}^{0}\right\|_{C^{0}}
\end{aligned}
$$

for all $t \in[0, T]$. As a consequence, the sequence $\left(V_{\mathrm{T}}^{n}\right)_{n \in \mathbb{N}}$ converges, uniformly on $[0, T]$, towards some limit $V_{\mathrm{T}} \in C^{0}\left([0, T] ; \mathbb{R}^{n-1}\right)$. Now, let $W_{\mathrm{T}} \in W^{1,1}\left([0, T] ; \mathbb{R}^{n-1}\right)$ be the solution of the evolution problem:

- $W_{\mathrm{T}}(0)=V_{0 \mathrm{~T}} ;$

- $\dot{W}_{\mathrm{T}}(t)+K_{\mathrm{T}} \cdot\left(U_{0 \mathrm{~T}}+\int_{0}^{t} V_{\mathrm{T}}(s) \mathrm{d} s\right)-F_{\mathrm{T}}(t) \in \partial S_{r \mathcal{B}}\left[-W_{\mathrm{T}}(t)\right], \quad$ for a.a. $t$.

Now, taking the difference of this differential inclusion with the one defining $V_{\mathrm{T}}^{n}$, multiplying by $W_{\mathrm{T}}-V_{\mathrm{T}}^{n}$ and integrating, we get, thanks to the monotonicity of the subdifferential and to Lemma A.5, p. 157 of [2]:

$$
\left|W_{\mathrm{T}}(t)-V_{\mathrm{T}}^{n}(t)\right| \leq\left|K_{\mathrm{T}}\right| T \int_{0}^{t}\left|V_{\mathrm{T}}(s)-V_{\mathrm{T}}^{n-1}(s)\right|
$$

for all $t \in[0, T]$, which shows that the sequence $\left(V_{\mathrm{T}}^{n}\right)_{n \in \mathbb{N}}$ converges, uniformly on $[0, T]$, towards $W_{\mathrm{T}}$. Hence, $V_{\mathrm{T}}=W_{\mathrm{T}} \in W^{1,1}$ and we have:

- $V_{\mathrm{T}}(0)=V_{0 \mathrm{~T}}$

- $\quad \dot{V}_{\mathrm{T}}(t)+K_{\mathrm{T}} \cdot\left(U_{0 \mathrm{~T}}+\int_{0}^{t} V_{\mathrm{T}}(s) \mathrm{d} s\right)-F_{\mathrm{T}}(t) \in \partial S_{r \mathcal{B}}\left[-V_{\mathrm{T}}(t)\right]$, for a.a. $t$.

Setting:

$$
U_{\mathrm{T}}(t)=U_{0 \mathrm{~T}}+\int_{0}^{t} V_{\mathrm{T}}(s) \mathrm{d} s
$$

we see that $U_{\mathrm{T}} \in W^{2,1}\left([0, T] ; \mathbb{R}^{n-1}\right)$ and provides the solution we sought.

Proposition 3.2. Let $r \in L^{1}([0, T] ; \mathbb{R})$ be a nonnegative integrable function and assume $F_{\mathrm{T}} \in L^{1}\left([0, T] ; \mathbb{R}^{n-1}\right)$. Then, there exists a unique $U_{\mathrm{T}} \in W^{2,1}\left([0, T] ; \mathbb{R}^{n-1}\right)$ such that:

- $U_{\mathrm{T}}(0)=U_{0 \mathrm{~T}} ; \quad \dot{U}_{\mathrm{T}}(0)=V_{0 \mathrm{~T}} \quad$ (initial condition);

- $\ddot{U}_{\mathrm{T}}(t)+K_{\mathrm{T}} \cdot U_{\mathrm{T}}(t)-F_{\mathrm{T}}(t) \in \partial S_{r(t) \cdot \mathcal{B}}\left[-\dot{U}_{\mathrm{T}}(t)\right], \quad$ for a.a. $t \in[0, T]$. 
Proof.

Uniqueness. Straightforward by monotonicity of the subdifferential.

Existence. Let $\left(r^{n}\right)_{n \in \mathbb{N}}$ be a sequence of nonnegative step functions on $[0, T]$, converging towards $r$ in $L^{1}([0, T] ; \mathbb{R})$. By Proposition 3.1, there exists a unique solution $U_{\mathrm{T}}^{n} \in W^{2,1}\left([0, T] ; \mathbb{R}^{n-1}\right)$ of:

- $U_{\mathrm{T}}^{n}(0)=U_{0 \mathrm{~T}} ; \quad \dot{U}_{\mathrm{T}}^{n}(0)=V_{0 \mathrm{~T}} \quad($ initial condition $) ;$

- $\ddot{U}_{\mathrm{T}}^{n}(t)+K_{\mathrm{T}} \cdot U_{\mathrm{T}}^{n}(t)-F_{\mathrm{T}}(t) \in \partial S_{r^{n}(t) \mathcal{B}}\left[-\dot{U}_{\mathrm{T}}^{n}(t)\right], \quad$ for a.a. $t \in[0, T]$.

Step 1. The sequences $\left(U_{\mathrm{T}}^{n}\right)_{n \in \mathbb{N}}$ and $\left(\dot{U}_{\mathrm{T}}^{n}\right)_{n \in \mathbb{N}}$ are Cauchy sequences in $C^{0}$ and, then, converge towards some limits $U_{\mathrm{T}}$ and $\dot{U}_{\mathrm{T}}$ in $C^{0}$.

Indeed:

$$
\begin{aligned}
\frac{1}{2}\left|\dot{U}_{\mathrm{T}}^{n+p}(t)-\dot{U}_{\mathrm{T}}^{n}(t)\right|^{2}+\frac{1}{2}\left[U_{\mathrm{T}}^{n+p}(t)-U_{\mathrm{T}}^{n}(t)\right] \cdot K_{\mathrm{T}} \cdot\left[U_{\mathrm{T}}^{n+p}(t)-U_{\mathrm{T}}^{n}(t)\right] & \leq-\int_{0}^{t}\left(r^{n+p}-r^{n}\right)\left(\left|\dot{U}_{\mathrm{T}}^{n+p}\right|-\left|\dot{U}_{\mathrm{T}}^{n}\right|\right) \\
& \leq \int_{0}^{t}\left|r^{n+p}-r^{n}\right| \cdot\left|\dot{U}_{\mathrm{T}}^{n+p}-\dot{U}_{\mathrm{T}}^{n}(t)\right|
\end{aligned}
$$

The conclusion follows by use of Lemma A.5, p. 157 of [2].

Step 2. $\dot{U}_{\mathrm{T}} \in W^{1,1}$.

Pick $0 \leq t_{1} \leq t_{2} \leq t$. Multiplying differential inclusion (1) by $\dot{U}_{\mathrm{T}}^{n}(s)-\dot{U}_{\mathrm{T}}^{n}\left(t_{1}\right)$ and integrating over $\left[t_{1}, t\right]$, we get:

$$
\begin{aligned}
& \frac{1}{2}\left|\dot{U}_{\mathrm{T}}^{n}(t)-\dot{U}_{\mathrm{T}}^{n}\left(t_{1}\right)\right|^{2}+\frac{1}{2}\left|U_{\mathrm{T}}^{n}(t)-U_{\mathrm{T}}^{n}\left(t_{1}\right)-\left(t-t_{1}\right) \dot{U}_{\mathrm{T}}^{n}\left(t_{1}\right)\right|_{K_{\mathrm{T}}}^{2} \\
& \quad \leq \int_{t_{1}}^{t}\left|F_{\mathrm{T}}(s)-K_{\mathrm{T}} \cdot\left[U_{\mathrm{T}}^{n}\left(t_{1}\right)+\left(s-t_{1}\right) \dot{U}_{\mathrm{T}}^{n}\left(t_{1}\right)\right]\right| \cdot\left|\dot{U}_{\mathrm{T}}^{n}(s)-\dot{U}_{\mathrm{T}}^{n}\left(t_{1}\right)\right| \mathrm{d} s+\int_{t_{1}}^{t} r^{n}(s)\left|\dot{U}_{\mathrm{T}}^{n}(s)-\dot{U}_{\mathrm{T}}^{n}\left(t_{1}\right)\right| \mathrm{d} s
\end{aligned}
$$

where $|\cdot|_{K_{\mathrm{T}}}$ denotes the norm on $\mathbb{R}^{n-1}$ which is associated with the scalar product defined by the symmetric positive definite matrix $K_{\mathrm{T}}$. Using, once more, Lemma A.5, p. 157 of [2], we obtain:

$$
\left|\dot{U}_{\mathrm{T}}^{n}\left(t_{2}\right)-\dot{U}_{\mathrm{T}}^{n}\left(t_{1}\right)\right| \leq \int_{t_{1}}^{t_{2}}\left[\left|F_{\mathrm{T}}(s)\right|+r^{n}(s)+M\right] \mathrm{d} s,
$$

where $M$ is some real constant, independent on $t_{1}, t_{2}$ and $n$. Taking the limit $n \rightarrow \infty$, we get:

$$
\left|\dot{U}_{\mathrm{T}}\left(t_{2}\right)-\dot{U}_{\mathrm{T}}\left(t_{1}\right)\right| \leq \int_{t_{1}}^{t_{2}}\left[\left|F_{\mathrm{T}}(s)\right|+r(s)+M\right] \mathrm{d} s,
$$

which shows that $\dot{U}_{\mathrm{T}}$ is absolutely continuous.

Step 3. $U_{\mathrm{T}}$ is a solution of the evolution problem under consideration.

Inequality (2) gives:

$$
\left\|\ddot{U}_{\mathrm{T}}^{n}\right\|_{L^{1}} \leq M T+\left\|r^{n}\right\|_{L^{1}}+\left\|F_{\mathrm{T}}\right\|_{L^{1}} \leq M^{\prime},
$$

where $M^{\prime}$ is a real constant independent of $n$. Therefore, extracting a subsequence if necessary, the sequence $\left(\ddot{U}_{\mathrm{T}}^{n}\right)_{n \in \mathbb{N}}$ converges in $\mathcal{M}\left([0, T] ; \mathbb{R}^{n-1}\right)$ weak-*. Its limit is necessarily $\ddot{U}_{\mathrm{T}}$. Now, $U_{\mathrm{T}}^{n}$ being a solution of evolution 
problem (1), we have:

$\forall V \in C^{0}\left([0, T] ; \mathbb{R}^{n-1}\right)$,

$$
\int_{0}^{T} r^{n}(s)|V(s)| \mathrm{d} s \geq \int_{0}^{T} r^{n}(s)\left|\dot{U}_{\mathrm{T}}^{n}(s)\right| \mathrm{d} s+\int_{0}^{T}\left[\ddot{U}_{\mathrm{T}}^{n}(s)+K_{\mathrm{T}} \cdot U_{\mathrm{T}}^{n}(s)-F_{\mathrm{T}}(s)\right]\left[V(s)+\dot{U}_{\mathrm{T}}^{n}(s)\right] \mathrm{d} s .
$$

Thanks to the convergence properties of all the sequences involved, we can take the limit as $n \rightarrow \infty$ in this inequality. We deduce that $U_{\mathrm{T}}$ is a solution of the evolution problem under consideration.

Proposition 3.3. Let $W \in \mathbb{R}^{n-1}, F_{\mathrm{N}} \in L^{1}([0, T] ; \mathbb{R})$ and $F_{\mathrm{T}} \in L^{1}\left([0, T] ; \mathbb{R}^{n-1}\right)$. Then, there exists a unique $U_{\mathrm{T}} \in W^{2,1}\left([0, T] ; \mathbb{R}^{n-1}\right)$ such that:

- $U_{\mathrm{T}}(0)=U_{0 \mathrm{~T}} ; \quad \dot{U}_{\mathrm{T}}(0)=V_{0 \mathrm{~T}} \quad$ (initial condition);

- $\ddot{U}_{\mathrm{T}}(t)+K_{\mathrm{T}} \cdot U_{\mathrm{T}}(t)-F_{\mathrm{T}}(t) \in \partial S_{\mu\left|F_{\mathrm{N}}(t)-W \cdot U_{\mathrm{T}}(t)\right| \cdot \mathcal{B}}\left[-\dot{U}_{\mathrm{T}}(t)\right], \quad$ for a.a. $t \in[0, T]$.

Proof.

Uniqueness. If $U_{\mathrm{T}}^{1}$ and $U_{\mathrm{T}}^{2}$ denote two solutions, then we have:

$$
\begin{aligned}
\frac{1}{2}\left|\dot{U}_{\mathrm{T}}^{1}(t)-\dot{U}_{\mathrm{T}}^{2}(t)\right|^{2}+\frac{1}{2}\left|U_{\mathrm{T}}^{1}(t)-U_{\mathrm{T}}^{2}(t)\right|_{K_{\mathrm{T}}}^{2} & \leq \mu \int_{0}^{t}\left|W \cdot U_{\mathrm{T}}^{1}(s)-W \cdot U_{\mathrm{T}}^{2}(s)\right| \cdot\left|\dot{U}_{\mathrm{T}}^{1}(s)-\dot{U}_{\mathrm{T}}^{2}(s)\right| \mathrm{d} s \\
& \leq \frac{\mu|W|}{2} \int_{0}^{t}\left(\left|\dot{U}_{\mathrm{T}}^{1}(s)-\dot{U}_{\mathrm{T}}^{2}(s)\right|^{2}+\left|U_{\mathrm{T}}^{1}(s)-U_{\mathrm{T}}^{2}(s)\right|^{2}\right) \mathrm{d} s
\end{aligned}
$$

and, therefore, $U_{\mathrm{T}}^{1} \equiv U_{\mathrm{T}}^{2}$, by Gronwall's lemma.

Existence. Let $U_{\mathrm{T}}^{n}$ be the sequence of functions in $W^{2,1}\left([0, T] ; \mathbb{R}^{n-1}\right)$ defined by:

$$
U_{\mathrm{T}}^{0}(t)=U_{0 \mathrm{~T}}+V_{0 \mathrm{~T}} t
$$

and by the following induction: knowing the function $U_{\mathrm{T}}^{n} \in W^{2,1}\left([0, T] ; \mathbb{R}^{n-1}\right), U_{\mathrm{T}}^{n+1}$ is the unique solution in $W^{2,1}\left([0, T] ; \mathbb{R}^{n-1}\right)$, provided by Proposition 3.2 , of the evolution problem:

- $U_{\mathrm{T}}^{n+1}(0)=U_{0 \mathrm{~T}} ; \quad \dot{U}_{\mathrm{T}}^{n+1}(0)=V_{0 \mathrm{~T}} \quad$ (initial condition);

- $\ddot{U}_{\mathrm{T}}^{n+1}(t)+K_{\mathrm{T}} \cdot U_{\mathrm{T}}^{n+1}(t)-F_{\mathrm{T}}(t) \in \partial S_{\mu\left|F_{\mathrm{N}}(t)-W \cdot U_{\mathrm{T}}^{n}(t)\right| \cdot \mathcal{B}}\left[-\dot{U}_{\mathrm{T}}^{n+1}(t)\right], \quad$ for a.a. $t$.

First, we get:

$$
\frac{1}{2}\left|\dot{U}_{\mathrm{T}}^{n+1}(t)-\dot{U}_{\mathrm{T}}^{n}(t)\right|^{2}+\frac{1}{2}\left|U_{\mathrm{T}}^{n+1}(t)-U_{\mathrm{T}}^{n}(t)\right|_{K_{\mathrm{T}}}^{2} \leq \mu|W| \int_{0}^{t}\left|U_{\mathrm{T}}^{n}(s)-U_{\mathrm{T}}^{n-1}(s)\right| \cdot\left|\dot{U}_{\mathrm{T}}^{n+1}(s)-\dot{U}_{\mathrm{T}}^{n}(s)\right| \mathrm{d} s
$$

and then, by Lemma A.5, p. 157 of [2]:

$$
\left|U_{\mathrm{T}}^{n+1}(t)-U_{\mathrm{T}}^{n}(t)\right| \leq C \int_{0}^{t}\left|U_{\mathrm{T}}^{n}(s)-U_{\mathrm{T}}^{n-1}(s)\right| \mathrm{d} s,
$$

where $C$ is a real constant independent of $t$ and $n$. Reusing the argument in the proof of Proposition 3.1, we obtain first the uniform convergence of the sequence $\left(U_{\mathrm{T}}^{n}\right)_{n \in \mathbb{N}}$, and then, coming back to inequality (3), the uniform convergence of the sequence $\left(\dot{U}_{\mathrm{T}}^{n}\right)_{n \in \mathbb{N}}$. Then, it can be shown, exactly as in the proof of Proposition 3.1, that this limit provides the solution we sought. 


\subsection{The bilateral problem with analytic force}

The aim of this section is to prove that, if the external force $F$ is not only integrable but analytic, then the solution of the bilateral problem $\mathcal{P}_{\mathrm{b}}$ provided by Proposition 3.3 is analytic on some right-neighbourhood of $t=0$.

Lemma 3.4. Let $n$ be a positive integer, $\mathcal{O}$ a neighbourhood of $(0,0)$ in $\mathbb{R}^{n} \times \mathbb{R}, G: \mathcal{O} \rightarrow \mathbb{R}^{n}$ an analytic function and $A$ a real square matrix of order $n$ without any eigenvalue in $\mathbb{N} \backslash\{0\}$. Then, there exist $\eta>0$ and an analytic function $X:\left[0, \eta\left[\rightarrow \mathbb{R}^{n}\right.\right.$ which is a solution of the Cauchy problem:

$$
\begin{aligned}
\text { - } X(0) & =0 \\
\text { - } \quad \dot{X}(t) & \left.=\frac{1}{t} A \cdot X(t)+G(X(t), t), \quad \forall t \in\right] 0, \eta[.
\end{aligned}
$$

Moreover, any other analytic solution of this Cauchy problem is, either a restriction, or an analytic extension of $X(t)$.

Proof. For the sake of clarity, the proof is presented only in the particular case $n=1$. For $|X|<r$ and $|t|<r$, we can write:

$$
G(X, t)=\sum_{i, j=0}^{\infty} g_{i j} X^{i} t^{j}
$$

Then, for $|X|<r$ and $|t|<r$, set:

$$
\widetilde{G}(X, t)=\sum_{i, j=0}^{\infty}\left|g_{i j}\right| X^{i} t^{j},
$$

and consider the Cauchy problem:

$$
\begin{aligned}
& \text { - } \widetilde{X}(0)=0 \text {; } \\
& \text { - } \frac{\mathrm{d}}{\mathrm{d} t} \widetilde{X}(t)=\widetilde{G}(\widetilde{X}(t), t), \quad \forall t,
\end{aligned}
$$

that admits a unique local solution $\widetilde{X}$ which, moreover, is analytic (Th. 1, p. 214 of [3]). This solution can be expanded in a power series:

$$
\widetilde{X}(t)=\sum_{i=1}^{\infty} \tilde{x}_{i} t^{i}
$$

which converges in a neighbourhood of $t=0$. The coefficients $\tilde{x}_{i}$ are inductively computed by substituting the power series expansion into the differential equation. This procedure gives, for all $k \in \mathbb{N}$ :

$$
(k+1) \tilde{x}_{k+1}=P_{k+1}\left(\tilde{x}_{1}, \tilde{x}_{2}, \ldots, \tilde{x}_{k} ;\left|g_{i j}\right|\right)
$$

where $P_{k+1}$ is a polynomial with integer coefficients, and arguments $\tilde{x}_{1}, \tilde{x}_{2}, \ldots, \tilde{x}_{k}$ and a finite number of $\left|g_{i j}\right|$. An induction argument based on equation (4) shows that all the $\tilde{x}_{i}$ are uniquely determined and satisfy in addition:

$$
\forall k \in \mathbb{N}, \quad \tilde{x}_{k+1} \geq 0 .
$$

Note that all the polynomials $P_{k+1}$ have the property:

$$
\forall \alpha \geq 1, \quad \forall \tilde{x}_{i} \in \mathbb{R}, \quad\left|P_{k+1}\left(\alpha \tilde{x}_{1}, \alpha^{2} \tilde{x}_{2}, \ldots, \alpha^{k} \tilde{x}_{k} ;\left|g_{i j}\right|\right)\right| \leq \alpha^{k} P_{k+1}\left(\left|\tilde{x}_{1}\right|,\left|\tilde{x}_{2}\right|, \ldots,\left|\tilde{x}_{k}\right| ;\left|g_{i j}\right|\right) .
$$


Now, let us come back to the Cauchy problem:

- $X(0)=0$;

- $\dot{X}(t)=\frac{1}{t} A X(t)+G(X(t), t)$,

and search a solution as a formal power series:

$$
X(t)=\sum_{i=1}^{\infty} x_{i} t^{i}
$$

Substituting this expression into the differential equation, we obtain, for all $k \in \mathbb{N}$ :

$$
(k+1-A) x_{k+1}=P_{k+1}\left(x_{1}, x_{2}, \ldots, x_{k} ; g_{i j}\right) \text {. }
$$

Set:

$$
\alpha=\sup _{k \in \mathbb{N}}\left\{\frac{k+1}{k+1-A}\right\} \quad(\alpha \geq 1),
$$

which is finite, since, by hypothesis, $A$ is not a positive integer. By virtue of the induction equation (6), we have, for all $k \in \mathbb{N}$ :

$$
\left|x_{k+1}\right| \leq \frac{\alpha}{k+1} P_{k+1}\left(\left|x_{1}\right|,\left|x_{2}\right|, \ldots,\left|x_{k}\right| ;\left|g_{i j}\right|\right) .
$$

By induction based on property (5), we get:

$$
\forall k \in \mathbb{N}, \quad\left|x_{k+1}\right| \leq \alpha^{k+1} \tilde{x}_{k+1},
$$

which proves that the convergence radius of the power series $\sum_{i \geq 1} x_{i} t^{i}$ is positive and, thus, gives the desired conclusion.

In the case where $n$ is arbitrary, the argument is similar, using the maximum norm on $\mathbb{R}^{n}$ instead of the absolute value; the constant $\alpha$ is then defined by:

$$
\alpha=\sup _{k \in \mathbb{N}}\left\{(k+1)\left|[(k+1) I-A]^{-1}\right|_{\infty}\right\} \quad(\alpha \geq 1)
$$

where $I$ is the identity matrix and $|\cdot|_{\infty}$ denotes the matrix norm associated with the maximum norm on $\mathbb{R}^{n}$.

Proposition 3.5. Let $F_{\mathrm{N}}:[0, T] \rightarrow \mathbb{R}$ and $F_{\mathrm{T}}:[0, T] \rightarrow \mathbb{R}^{n-1}$ be two analytic functions. Then, there exists $\eta>0$ such that the restriction to $\left[0, \eta\left[\right.\right.$ of $U_{\mathrm{T}} \in W^{2,1}$, provided by Proposition 3.3, is analytic.

Proof. By the assumed analyticity of functions $F_{\mathrm{N}}(t)$ and $F_{\mathrm{T}}(t)$, there exists $\eta>0$ such that, necessarily, one of the following three cases occurs.

Case 1. $V_{0 \mathrm{~T}} \neq 0$.

Case 2. $V_{0 \mathrm{~T}}=0$ and $\left.\forall t \in\right] 0, \eta\left[, \quad\left|F_{\mathrm{T}}(t)-K_{\mathrm{T}} \cdot U_{0 \mathrm{~T}}\right| \leq \mu\left|F_{\mathrm{N}}(t)-W \cdot U_{0 \mathrm{~T}}\right|\right.$.

Case 3. $V_{0 \mathrm{~T}}=0$ and $\left.\forall t \in\right] 0, \eta\left[, \quad\left|F_{\mathrm{T}}(t)-K_{\mathrm{T}} \cdot U_{0 \mathrm{~T}}\right|>\mu\left|F_{\mathrm{N}}(t)-W \cdot U_{0 \mathrm{~T}}\right|\right.$.

Thus, we are going to prove that the conclusion is reached in any of these cases.

Case 1. $V_{0 \mathrm{~T}} \neq 0$.

Let $\mathcal{O}$ be an open neighbourhood of $V_{0 \mathrm{~T}}$ in $\mathbb{R}^{n-1}$ which does not contain 0 . Then, the function:

$$
\left\{\begin{array}{l}
\mathcal{O} \rightarrow \mathbb{R}^{n-1} \\
V \mapsto \frac{V}{|V|}
\end{array}\right.
$$


is analytic. Cauchy-Lipschitz' theorem provides a solution $U_{\mathrm{T}} \in C^{2}\left(\left[0, \alpha\left[; \mathbb{R}^{n-1}\right)\right.\right.$ of the Cauchy problem:

- $U_{\mathrm{T}}(0)=U_{0 \mathrm{~T}} ; \quad \dot{U}_{\mathrm{T}}(0)=V_{0 \mathrm{~T}} ;$

- $\ddot{U}_{\mathrm{T}}(t)+K_{\mathrm{T}} \cdot U_{\mathrm{T}}(t)+\mu\left|F_{\mathrm{N}}(t)-W \cdot U_{\mathrm{T}}(t)\right| \frac{\dot{U}_{\mathrm{T}}(t)}{\left|\dot{U}_{\mathrm{T}}(t)\right|}=F_{\mathrm{T}}(t)$;

- $\dot{U}_{\mathrm{T}}(t) \in \mathcal{O}$.

Now, seeking a solution of (7) as a formal power series:

$$
U_{\mathrm{T}}(t)=\sum_{k=0}^{\infty} \lambda_{k} t^{k}
$$

and substituting into (7), we have necessarily:

$$
\lambda_{0}=U_{0 \mathrm{~T}}, \quad \lambda_{1}=V_{0 \mathrm{~T}}
$$

and then:

$$
\lambda_{2}=\frac{1}{2}\left\{F_{\mathrm{T}}(0)-K_{\mathrm{T}} \cdot U_{0 \mathrm{~T}}-\left|F_{\mathrm{N}}(0)-W \cdot U_{0 \mathrm{~T}}\right| \frac{V_{0 \mathrm{~T}}}{\left|V_{0 \mathrm{~T}}\right|}\right\} .
$$

Replacing the term $\left|F_{\mathrm{N}}(t)-W \cdot U_{\mathrm{T}}(t)\right|$, in $(7)$, by $\pm\left[F_{\mathrm{N}}(t)-W \cdot U_{\mathrm{T}}(t)\right]$ according to the sign of the first nonzero term of the formal power series expansion of $\left[F_{\mathrm{N}}(t)-W \cdot U_{\mathrm{T}}(t)\right]$, it is readily seen, by induction, that the sequence $\left(\lambda_{n}\right)_{n \in \mathbb{N}}$ is uniquely determined. Only two cases are possible.

Case 1.1. $\forall n \in \mathbb{N}, \quad \frac{F_{\mathrm{N}}^{(n)}(0)}{n !}=W \cdot \lambda_{n}$, in which case the solution of the Cauchy problem:

- $U_{\mathrm{T}}(0)=U_{0 \mathrm{~T}} ; \quad \dot{U}_{\mathrm{T}}(0)=V_{0 \mathrm{~T}} ;$

- $\ddot{U}_{\mathrm{T}}(t)+K_{\mathrm{T}} \cdot U_{\mathrm{T}}(t)=F_{\mathrm{T}}(t)$;

- $\dot{U}_{\mathrm{T}}(t) \in \mathcal{O}$,

which is analytic by Theorem 1, p. 214 of [3], has the $\lambda_{n}$ as coefficients of its power series expansion at 0 . Hence, the $\lambda_{n}$ are the coefficients of the power series expansion at 0 of a certain analytic function defined on a right-neighbourhood of 0 and which solves problem (7) and therefore the Cauchy problem of Proposition 3.3. Case 1.2. $\forall n \in\left\{0,1, \ldots, n_{0}-1\right\}, \quad \frac{F_{\mathrm{N}}^{(n)}(0)}{n !}=W \cdot \lambda_{n}, \quad$ and $\quad \frac{F_{\mathrm{N}}^{\left(n_{0}\right)}(0)}{n_{0} !} \neq W \cdot \lambda_{n_{0}}$, in which case the analytic solution of the Cauchy problem:

- $U_{\mathrm{T}}(0)=U_{0 \mathrm{~T}} ; \quad \dot{U}_{\mathrm{T}}(0)=V_{0 \mathrm{~T}} ;$

- $\ddot{U}_{\mathrm{T}}(t)+K_{\mathrm{T}} \cdot U_{\mathrm{T}}(t)+\mu \operatorname{sgn}\left[\frac{F_{\mathrm{N}}^{\left(n_{0}\right)}(0)}{n_{0} !}-W \cdot \lambda_{n_{0}}\right]\left[F_{\mathrm{N}}(t)-W \cdot U_{\mathrm{T}}(t)\right] \frac{\dot{U}_{\mathrm{T}}(t)}{\left|\dot{U}_{\mathrm{T}}(t)\right|}=F_{\mathrm{T}}(t)$;

- $\dot{U}_{\mathrm{T}}(t) \in \mathcal{O}$,

is a solution of problem (7) on a right-neighbourhood of $t=0$ and therefore solves the Cauchy problem of Proposition 3.3.

Case 2. $V_{0 \mathrm{~T}}=0 \quad$ and $\left.\forall t \in\right] 0, \eta\left[, \quad\left|F_{\mathrm{T}}(t)-K_{\mathrm{T}} \cdot U_{0 \mathrm{~T}}\right| \leq \mu\left|F_{\mathrm{N}}(t)-W \cdot U_{0 \mathrm{~T}}\right|\right.$. 
In this case, it is readily checked that the constant function $U_{\mathrm{T}} \equiv U_{0 \mathrm{~T}}$ on $[0, \eta[$ (which is analytic) provides a solution on $[0, \eta[$ of the Cauchy problem of Proposition 3.3.

Case 3. $V_{0 \mathrm{~T}}=0$ and $\left.\forall t \in\right] 0, \eta\left[, \quad\left|F_{\mathrm{T}}(t)-K_{\mathrm{T}} \cdot U_{0 \mathrm{~T}}\right|>\mu\left|F_{\mathrm{N}}(t)-W \cdot U_{0 \mathrm{~T}}\right|\right.$.

This case is the most tricky to examine. Our technique will consist to transform problem (7) into a form on which Lemma 3.4 applies.

By the assumed analyticity of functions $F_{\mathrm{N}}(t)$ and $F_{\mathrm{T}}(t)$, together with the hypothesis of case 3 , we know that there exist two integers $n_{0}$ and $n_{1} \geq n_{0}$ such that:

$$
\begin{array}{cl}
F_{\mathrm{T}}(t)-K_{\mathrm{T}} \cdot U_{0 \mathrm{~T}} \sim \alpha t^{n_{0}}, & \alpha \in \mathbb{R}^{n-1} \backslash\{0\}, \\
F_{\mathrm{N}}(t)-W \cdot U_{0 \mathrm{~T}} \sim \beta t^{n_{1}}, & \beta \in \mathbb{R} \backslash\{0\},
\end{array}
$$

when $t$ goes to 0 (in case where $F_{\mathrm{N}}(t) \equiv W \cdot U_{0 \mathrm{~T}}$, just set $n_{1}=+\infty$ in the sequel). Let us look for a formal power series solution of problem (7). It is readily checked that the first nonzero term of the formal power series associated to $U_{\mathrm{T}}-U_{0 \mathrm{~T}}$ can be written:

$$
\gamma t^{n_{0}+2}
$$

where $\gamma$ must satisfy the equation:

$$
\left(n_{0}+2\right)\left(n_{0}+1\right) \gamma+\mu \delta_{n_{0}}^{n_{1}}|\beta| \frac{\gamma}{|\gamma|}=\alpha .
$$

Here, $\delta_{n_{0}}^{n_{1}}$ denotes the Kronecker index (which equals 1 , if $n_{0}=n_{1}$, and 0 , otherwise). The solution of equation (8) is:

Then, we define new unknown functions, for $t>0$, by:

$$
\gamma=\frac{|\alpha|-\mu \delta_{n_{0}}^{n_{1}}|\beta|}{\left(n_{0}+2\right)\left(n_{0}+1\right)} \cdot \frac{\alpha}{|\alpha|}
$$

$$
\begin{aligned}
& \widetilde{U}_{\mathrm{T}}=\frac{U_{\mathrm{T}}-U_{0 \mathrm{~T}}}{t^{n_{0}+1}}, \\
& \widetilde{V}_{\mathrm{T}}=\frac{\dot{U}_{\mathrm{T}}}{\left(n_{0}+2\right) t^{n_{0}+1}}-\gamma .
\end{aligned}
$$

Hence, for $t>0$, the functions $\widetilde{U}_{\mathrm{T}}$ and $\widetilde{V}_{\mathrm{T}}$ are related by the differential equation:

$$
\frac{\mathrm{d}}{\mathrm{d} t} \widetilde{U}_{\mathrm{T}}=-\frac{n_{0}+1}{t} \widetilde{U}_{\mathrm{T}}+\left(n_{0}+2\right)\left(\widetilde{V}_{\mathrm{T}}+\gamma\right)
$$

Now, there remains to write the differential equation in problem (7) in terms of the new unknown functions $\widetilde{U}_{\mathrm{T}}$ and $\widetilde{V}_{\mathrm{T}}$. We get:

$$
\frac{\mathrm{d}}{\mathrm{d} t} \widetilde{V}_{\mathrm{T}}=-\frac{n_{0}+1}{t}\left(\widetilde{V}_{\mathrm{T}}+\gamma\right)-\frac{1}{n_{0}+2} K_{\mathrm{T}} \cdot \widetilde{U}_{\mathrm{T}}+\frac{F_{\mathrm{T}}-K_{\mathrm{T}} \cdot U_{0 \mathrm{~T}}}{\left(n_{0}+2\right) t^{n_{0}+1}}-\frac{\mu}{n_{0}+2}\left|\frac{F_{\mathrm{N}}-W \cdot U_{0 \mathrm{~T}}}{t^{n_{0}+1}}-W \cdot \widetilde{U}_{\mathrm{T}}\right| \frac{\gamma+\widetilde{V}_{\mathrm{T}}}{\left|\gamma+\widetilde{V}_{\mathrm{T}}\right|},
$$

which is, using definition (8) of $\gamma$, nothing but:

$$
\begin{aligned}
\frac{\mathrm{d}}{\mathrm{d} t} \widetilde{V}_{\mathrm{T}}=-\frac{n_{0}+1}{t} \widetilde{V}_{\mathrm{T}}-\frac{1}{n_{0}+2} K_{\mathrm{T}} \cdot \widetilde{U}_{\mathrm{T}}+\frac{F_{\mathrm{T}}-K_{\mathrm{T}} \cdot U_{0 \mathrm{~T}}-\alpha t^{n_{0}}}{\left(n_{0}+2\right) t^{n_{0}+1}} & \\
& +\frac{\mu \delta_{n_{0}}^{n_{1}}|\beta|}{\left(n_{0}+2\right) t} \cdot \frac{\gamma}{|\gamma|}-\frac{\mu}{n_{0}+2}\left|\frac{F_{\mathrm{N}}-W \cdot U_{0 \mathrm{~T}}}{t^{n_{0}+1}}-W \cdot \widetilde{U}_{\mathrm{T}}\right| \frac{\gamma+\widetilde{V}_{\mathrm{T}}}{\left|\gamma+\widetilde{V}_{\mathrm{T}}\right|} .
\end{aligned}
$$


Now, it is readily seen that the Cauchy problem:

- $\widetilde{U}_{\mathrm{T}}(0)=0 ; \quad \widetilde{V}_{\mathrm{T}}(0)=0 ;$

- $\frac{\mathrm{d}}{\mathrm{d} t} \widetilde{U}_{\mathrm{T}}=-\frac{n_{0}+1}{t} \widetilde{U}_{\mathrm{T}}+\left(n_{0}+2\right)\left(\gamma+\widetilde{V}_{\mathrm{T}}\right)$;

- $\frac{\mathrm{d}}{\mathrm{d} t} \widetilde{V}_{\mathrm{T}}=-\frac{n_{0}+1}{t} \widetilde{V}_{\mathrm{T}}-\frac{1}{n_{0}+2} K_{\mathrm{T}} \cdot \widetilde{U}_{\mathrm{T}}+\frac{F_{\mathrm{T}}-K_{\mathrm{T}} \cdot U_{0 \mathrm{~T}}-\alpha t^{n_{0}}}{\left(n_{0}+2\right) t^{n_{0}+1}}$

$$
+\frac{\mu \delta_{n_{0}}^{n_{1}}|\beta|}{\left(n_{0}+2\right) t} \cdot \frac{\gamma}{|\gamma|}-\frac{\mu}{n_{0}+2}\left|\frac{F_{\mathrm{N}}-W \cdot U_{0 \mathrm{~T}}}{t^{n_{0}+1}}-W \cdot \widetilde{U}_{\mathrm{T}}\right| \frac{\gamma+\widetilde{V}_{\mathrm{T}}}{\left|\gamma+\widetilde{V}_{\mathrm{T}}\right|}
$$

has a unique formal power series solution. Let sign equal -1 or +1 according to the sign of the first nonzero term in the formal power series expansion of:

$$
F_{\mathrm{N}}-W \cdot U_{0 \mathrm{~T}}-t^{n_{0}+1} W \cdot \widetilde{U}_{\mathrm{T}}
$$

It is easily checked that, in the particular case $n_{1}=n_{0}$, we have:

$$
\operatorname{sign}=\operatorname{sgn}(\beta),
$$

so that the function $\widetilde{G}$ defined by:

$$
\widetilde{G}\left(\widetilde{U}_{\mathrm{T}}, \widetilde{V}_{\mathrm{T}}, t\right)=\frac{F_{\mathrm{T}}-K_{\mathrm{T}} \cdot U_{0 \mathrm{~T}}-\alpha t^{n_{0}}}{\left(n_{0}+2\right) t^{n_{0}+1}}+\frac{\mu \delta_{n_{0}}^{n_{1}}|\beta|}{\left(n_{0}+2\right) t} \cdot \frac{\gamma}{|\gamma|}-\frac{\mu \operatorname{sign}}{n_{0}+2}\left[\frac{F_{\mathrm{N}}-W \cdot U_{0 \mathrm{~T}}}{t^{n_{0}+1}}-W \cdot \widetilde{U}_{\mathrm{T}}\right] \frac{\gamma+\widetilde{V}_{\mathrm{T}}}{\left|\gamma+\widetilde{V}_{\mathrm{T}}\right|}
$$

is analytic on some neighbourhood of $(0,0,0)$. Then, Lemma 3.4 provides a local analytic solution $\left(\widetilde{U}_{\mathrm{T}}, \widetilde{V}_{\mathrm{T}}\right)$ of the problem:

- $\widetilde{U}_{\mathrm{T}}(0)=0 ; \quad \widetilde{V}_{\mathrm{T}}(0)=0 ;$

- $\frac{\mathrm{d}}{\mathrm{d} t} \widetilde{U}_{\mathrm{T}}=-\frac{n_{0}+1}{t} \widetilde{U}_{\mathrm{T}}+\left(n_{0}+2\right)\left(\gamma+\widetilde{V}_{\mathrm{T}}\right)$;

- $\frac{\mathrm{d}}{\mathrm{d} t} \widetilde{V}_{\mathrm{T}}=-\frac{n_{0}+1}{t} \widetilde{V}_{\mathrm{T}}-\frac{1}{n_{0}+2} K_{\mathrm{T}} \cdot \widetilde{U}_{\mathrm{T}}+\widetilde{G}\left(\widetilde{U}_{\mathrm{T}}, \widetilde{V}_{\mathrm{T}}, t\right)$.

Setting:

$$
U_{\mathrm{T}}(t)=U_{0 \mathrm{~T}}+t^{n_{0}+1} \widetilde{U}_{\mathrm{T}}(t),
$$

the function $U_{\mathrm{T}}$ is analytic on a right-neighbourhood of 0 and:

$$
\dot{U}_{\mathrm{T}}(t)=\left(n_{0}+2\right) t^{n_{0}+1}\left(\gamma+\widetilde{V}_{\mathrm{T}}(t)\right)
$$

Rewinding the argument, it is readily seen that $U_{\mathrm{T}}$ is a solution of problem (7) and therefore, of the evolution problem of Proposition 3.3.

\section{The Unilateral PROBlem With ANALYTiC FORCE}

\subsection{Existence of a local analytic solution}

The result announced in the title of this section is the following. 
Theorem 4.1. Let $F:[0, T] \rightarrow \mathbb{R}^{n}$ be an analytic function. Then, there exist $T_{\mathrm{a}}>0$ and analytic functions $U_{\mathrm{a}}:\left[0, T_{\mathrm{a}}\left[\rightarrow \mathbb{R}^{n}\right.\right.$ and $R_{\mathrm{aN}}:\left[0, T_{\mathrm{a}}[\rightarrow \mathbb{R}\right.$, solution of the problem:

- $U_{\mathrm{a}}(0)=U_{0} ; \quad \dot{U}_{\mathrm{a}}(0)=V_{0} ;$

- $\ddot{U}_{\mathrm{aN}}+k_{\mathrm{N}} U_{\mathrm{aN}}+W \cdot U_{\mathrm{aT}}=F_{\mathrm{N}}+R_{\mathrm{aN}}, \quad$ in $\left[0, T_{\mathrm{a}}[\right.$;

- $\ddot{U}_{\mathrm{aT}}+K_{\mathrm{T}} \cdot U_{\mathrm{aT}}+W U_{\mathrm{aN}}-F_{\mathrm{T}} \in \partial S_{-\mu R_{\mathrm{aN}} \cdot \mathcal{B}}\left[-\dot{U}_{\mathrm{aT}}\right], \quad$ in $\left[0, T_{\mathrm{a}}[\right.$;

- $U_{\mathrm{aN}} \leq 0, \quad R_{\mathrm{aN}} \leq 0, \quad U_{\mathrm{aN}} R_{\mathrm{aN}} \equiv 0$.

Moreover, any other analytic solution of this evolution problem is, either a restriction, or an analytic extension of this solution.

Proof. If we do not have $U_{0 \mathrm{~N}}=V_{0 \mathrm{~N}}=0$, Theorem 4.1 is obvious, so we concentrate on the case $U_{0 \mathrm{~N}}=V_{0 \mathrm{~N}}=0$. Denoting by:

$$
F(t)=\sum_{i=0}^{\infty} f_{i} t^{i}
$$

the power series expansion of $F$ at $t=0$, we shall look for a formal power series solution given by:

$$
U_{\mathrm{a}}=\sum_{i=2}^{\infty} u_{i} t^{i}, \quad R_{\mathrm{aN}}=\sum_{i=0}^{\infty} r_{i} t^{i} .
$$

The first terms of these two formal series must satisfy:

- $2 u_{2 \mathrm{~N}}=f_{0 \mathrm{~N}}+r_{0}$;

- $u_{2 \mathrm{~N}} \leq 0, \quad r_{0} \leq 0, \quad u_{2 \mathrm{~N}} r_{0}=0$.

This system determines uniquely the couple $\left(u_{2 \mathrm{~N}}, r_{0}\right)$. If this couple does not vanish, we stop. Otherwise, we continue the induction until, perhaps, a couple $\left(u_{(i+2) \mathrm{N}}, r_{i}\right)$ becomes distinct from $(0,0)$. At rank $i$, the problem to be solved is:

- $\quad i(i+1) u_{(i+1) \mathrm{T}}+K_{\mathrm{T}} u_{(i-1) \mathrm{T}}+W u_{(i-1) \mathrm{N}}=f_{(i-1) \mathrm{T}}$;

- $\quad(i+1)(i+2) u_{(i+2) \mathrm{N}}+k_{\mathrm{N}} u_{i \mathrm{~N}}+W \cdot u_{i \mathrm{~T}}=f_{i}+r_{i}$;

- $u_{(i+2) \mathrm{N}} \leq 0, \quad r_{i} \leq 0, \quad u_{(i+2) \mathrm{N}} r_{i}=0$.

The two following cases have to be considered.

Case 1. The induction does not stop because all the couples $\left(u_{(i+2) \mathrm{N}}, r_{i}\right)$ vanish.

Then, Theorem 1 , p. 214 of $[3]$ provides an analytic solution $u_{\mathrm{a}}:\left[0, T_{\mathrm{a}}\left[\rightarrow \mathbb{R}^{n}\right.\right.$ of the problem:

- $U_{\mathrm{a}}(0)=U_{0} ; \quad \dot{U}_{\mathrm{a}}(0)=V_{0} ;$

- $\ddot{U}_{\mathrm{a}}+K \cdot U_{\mathrm{a}}=F, \quad$ in $\left[0, T_{\mathrm{a}}[\right.$.

This solution, associated with the choice $R_{\mathrm{aN}} \equiv 0$ provides the sought analytic solution of the evolution problem under consideration.

Case 2. The induction stops at rank $n_{0}$ because $u_{\left(n_{0}+2\right) \mathrm{N}}<0$.

Then, Theorem 1, p. 214 of [3] provides an analytic solution $U_{\mathrm{a}}:\left[0, T_{\mathrm{a}}\left[\rightarrow \mathbb{R}^{n}\right.\right.$ of the problem:

- $U_{\mathrm{a}}(0)=U_{0} ; \quad \dot{U}_{\mathrm{a}}(0)=V_{0}$;

- $\ddot{U}_{\mathrm{a}}+K \cdot U_{\mathrm{a}}=F, \quad$ in $\left[0, T_{\mathrm{a}}[\right.$. 
Restricting, if necessary, the time interval on which $U_{\mathrm{a}}$ is defined, we have:

$$
\forall t \in] 0, T_{\mathrm{a}}\left[, \quad U_{\mathrm{aN}}(t)<0,\right.
$$

and this solution, associated with the choice $R_{\mathrm{aN}} \equiv 0$ provides the sought analytic solution of the evolution problem under consideration.

Case 3. The induction stops at rank $n_{0}$ because $r_{n_{0}}<0$.

Propositions 3.3 and 3.5 provide an analytic solution $U_{\mathrm{aT}}:\left[0, T_{\mathrm{a}}\left[\rightarrow \mathbb{R}^{n-1}\right.\right.$ of the problem:

$$
\begin{array}{ll}
\text { - } & U_{\mathrm{aT}}(0)=0 ; \quad \dot{U}_{\mathrm{aT}}(0)=0 \\
\text { - } & \ddot{U}_{\mathrm{aT}}(t)+K_{\mathrm{T}} \cdot U_{\mathrm{aT}}(t)-F_{\mathrm{T}}(t) \in \partial S_{\mu\left|F_{\mathrm{N}}(t)-W \cdot U_{\mathrm{aT}}(t)\right| \cdot \mathcal{B}}\left[-\dot{U}_{\mathrm{aT}}(t)\right], \quad \forall t \in\left[0, T_{\mathrm{a}}[.\right.
\end{array}
$$

Restricting, if necessary, the time interval on which $U_{\mathrm{aT}}$ is defined, we have:

$$
\forall t \in] 0, T_{\mathrm{a}}\left[, \quad W \cdot U_{\mathrm{aT}}(t)-F_{\mathrm{N}}(t)<0,\right.
$$

and this function, associated with the choices:

$$
U_{\mathrm{aN}} \equiv 0, \quad R_{\mathrm{aN}} \equiv W \cdot U_{\mathrm{aT}}-F_{\mathrm{N}},
$$

provides the sought analytic solution of the evolution problem under consideration.

The uniqueness part of the theorem comes from the fact that the induction (finite or infinite) determines the status (active contact or not) of the system on a right-neighbourhood of $t=0$, and uniqueness at fixed status holds either by virtue of Theorem 1, p. 214 of [3], or by virtue of Propositions 3.3 and 3.5.

\subsection{Local uniqueness for the unilateral problem with analytic force}

The result announced in the title of this section is the following.

Theorem 4.2. Let $F:[0, T] \rightarrow \mathbb{R}^{n}$ be an analytic function, $U_{\mathrm{a}}:\left[0, T_{\mathrm{a}}\left[\rightarrow \mathbb{R}^{n}\right.\right.$, the local analytic solution of problem $\mathcal{P}_{\mathrm{u}}$ provided by Theorem 4.1 and $U \in M M A\left([0, T] ; \mathbb{R}^{n}\right)$, an arbitrary solution of problem $\mathcal{P}_{\mathrm{u}}$. Then, $U_{\mathrm{a}}$ and $U$ are identically equal on some right-neighbourhood of $t=0$ :

$$
\exists T^{\prime} \leq T_{\mathrm{a}}, \quad \forall t \in\left[0, T^{\prime}\left[, \quad U_{\mathrm{a}}(t)=U(t) .\right.\right.
$$

Proof.

Step 1. For all $t \in\left[0, T_{\mathrm{a}}[\right.$, the following estimate holds:

$$
\left|\dot{U}_{\mathrm{T}}^{+}-\dot{U}_{\mathrm{aT}}\right|(t)+\left|U_{\mathrm{T}}-U_{\mathrm{aT}}\right|(t) \leq C_{1} \int_{[0, t]}\left|R_{\mathrm{N}}-R_{\mathrm{aN}}\right|+C_{2} \int_{0}^{t}\left|U_{\mathrm{N}}-U_{\mathrm{aN}}\right|,
$$

for some real constants $C_{1}$ and $C_{2}$ depending only on $K$ and $\mu$.

We start with:

$$
\ddot{U}_{\mathrm{T}}-\ddot{U}_{\mathrm{aT}}+K_{\mathrm{T}} \cdot\left(U_{\mathrm{T}}-U_{\mathrm{aT}}\right)+\left(U_{\mathrm{N}}-U_{\mathrm{aN}}\right) W=R_{\mathrm{T}}-R_{\mathrm{aT}} .
$$

We multiply by $\dot{U}_{\mathrm{T}}^{+}-\dot{U}_{\mathrm{aT}}$ and integrate over $[0, t]$. The Coulomb friction law gives:

$$
\begin{aligned}
\int_{[0, t]}\left(R_{\mathrm{T}}-R_{\mathrm{aT}}\right) \cdot\left(\dot{U}_{\mathrm{T}}^{+}-\dot{U}_{\mathrm{aT}}\right) & \leq \mu \int_{[0, t]}\left(R_{\mathrm{N}}-R_{\mathrm{aN}}\right)\left(\left|\dot{U}_{\mathrm{T}}^{+}\right|-\left|\dot{U}_{\mathrm{aT}}\right|\right) \\
& \leq \mu \int_{[0, t]}\left|R_{\mathrm{N}}-R_{\mathrm{aN}}\right|\left|\dot{U}_{\mathrm{T}}^{+}-\dot{U}_{\mathrm{aT}}\right|
\end{aligned}
$$


Moreover:

$$
\int_{[0, t]} \ddot{U}_{\mathrm{T}} \cdot \dot{U}_{\mathrm{T}}^{+} \geq \frac{1}{2}\left|\dot{U}_{\mathrm{T}}^{+}\right|^{2}(t)-\frac{1}{2}\left|V_{0 \mathrm{~T}}\right|^{2}
$$

(by use of [9], p. 44), which leads to:

$$
\int_{[0, t]}\left(\ddot{U}_{\mathrm{T}}-\ddot{U}_{\mathrm{aT}}\right) \cdot\left(\dot{U}_{\mathrm{T}}^{+}-\dot{U}_{\mathrm{aT}}\right) \geq \frac{1}{2}\left|\dot{U}_{\mathrm{T}}^{+}-\dot{U}_{\mathrm{aT}}\right|^{2}(t)
$$

Putting everything together, we get, for all $t \in\left[0, T_{\mathrm{a}}[\right.$ :

$$
\frac{1}{2}\left|\dot{U}_{\mathrm{T}}^{+}-\dot{U}_{\mathrm{aT}}\right|^{2}(t)+\frac{1}{2}\left|U_{\mathrm{T}}-U_{\mathrm{aT}}\right|_{K_{\mathrm{T}}}^{2}(t) \leq \mu \int_{[0, t]}\left|R_{\mathrm{N}}-R_{\mathrm{aN}}\right|\left|\dot{U}_{\mathrm{T}}^{+}-\dot{U}_{\mathrm{aT}}\right|+|W| \int_{0}^{t}\left|U_{\mathrm{N}}-U_{\mathrm{aN}}\right|\left|\dot{U}_{\mathrm{T}}^{+}-\dot{U}_{\mathrm{aT}}\right|
$$

Then, Lemma A.5, p. 157 of [2] gives the estimate we looked for:

$$
\left|\dot{U}_{\mathrm{T}}^{+}-\dot{U}_{\mathrm{aT}}\right|(t)+\left|U_{\mathrm{T}}-U_{\mathrm{aT}}\right|_{K_{\mathrm{T}}}(t) \leq \mu \sqrt{2} \int_{[0, t]}\left|R_{\mathrm{N}}-R_{\mathrm{aN}}\right|+|W| \sqrt{2} \int_{0}^{t}\left|U_{\mathrm{N}}-U_{\mathrm{aN}}\right|
$$

Step 2. For all $t \in\left[0, T_{\mathrm{a}}[\right.$, the following estimate holds:

$$
\left|\dot{U}_{\mathrm{N}}^{+}-\dot{U}_{\mathrm{aN}}\right|(t)+\left|U_{\mathrm{N}}-U_{\mathrm{aN}}\right|(t) \leq C_{3} \int_{0}^{t}\left|U_{\mathrm{T}}-U_{\mathrm{aT}}\right|+C_{4} \int_{0}^{t}\left|R_{\mathrm{aN}}\right|,
$$

for some real constants $C_{3}$ et $C_{4}$ depending only on $K$.

We start with:

$$
\ddot{U}_{\mathrm{N}}-\ddot{U}_{\mathrm{aN}}+k_{\mathrm{N}}\left(U_{\mathrm{N}}-U_{\mathrm{aN}}\right)+W \cdot\left(U_{\mathrm{T}}-U_{\mathrm{aT}}\right)=R_{\mathrm{N}}-R_{\mathrm{aN}} .
$$

We multiply by $\left(\dot{U}_{\mathrm{N}}^{+}+\dot{U}_{\mathrm{N}}^{-}\right) / 2-\dot{U}_{\mathrm{aN}}$ and integrate over $[0, t]$. We get:

$$
\frac{1}{2}\left|\dot{U}_{\mathrm{N}}^{+}-\dot{U}_{\mathrm{aN}}\right|^{2}(t)+\frac{k_{\mathrm{N}}}{2}\left|U_{\mathrm{N}}-U_{\mathrm{aN}}\right|^{2}(t)=\int_{[0, t]}\left(R_{\mathrm{N}}-R_{\mathrm{aN}}\right)\left(\left(\dot{U}_{\mathrm{N}}^{+}+\dot{U}_{\mathrm{N}}^{-}\right) / 2-\dot{U}_{\mathrm{aN}}\right)-\int_{0}^{t}\left(\dot{U}_{\mathrm{N}}^{+}-\dot{U}_{\mathrm{aN}}\right) W \cdot\left(U_{\mathrm{T}}-U_{\mathrm{aT}}\right) .
$$

But, note the two following remarks.

(1) Restricting, if necessary, the time interval we work on:

$$
R_{\mathrm{N}} \dot{U}_{\mathrm{aN}} \geq 0
$$

because, if $U_{0 \mathrm{~N}}<0$, then $R_{\mathrm{N}}$ must vanish on a right neighbourhood of $t=0$, and, if $U_{0 \mathrm{~N}}=0$, then the nonpositive analytic function $U_{\mathrm{aN}}$ must be nonincreasing on a right neighbourhood of $t=0$.

(2) The measure defined by:

$$
R_{\mathrm{N}}\left(\dot{U}_{\mathrm{N}}^{+}+\dot{U}_{\mathrm{N}}^{-}\right) \leq 0
$$

is nonpositive. Indeed, let $D$ be the countable subset of $[0, T]$ of those instants $t$ at which the normal velocity is discontinuous: $\dot{U}_{\mathrm{N}}^{+}(t) \neq \dot{U}_{\mathrm{N}}^{-}(t)$. On $[0, T] \backslash D$, the measure $R_{\mathrm{N}}\left(\dot{U}_{\mathrm{N}}^{+}+\dot{U}_{\mathrm{N}}^{-}\right)$equals $R_{\mathrm{N}} \dot{U}_{\mathrm{N}}^{-}$ which is nonpositive, thanks to the unilateral contact condition (actually, the measure $R_{\mathrm{N}} \dot{U}_{\mathrm{N}}^{-}$vanishes identically on $[0, T] \backslash D)$. Moreover, at each instant $t \in D$, the measure $R_{\mathrm{N}}\left(\dot{U}_{\mathrm{N}}^{+}+\dot{U}_{\mathrm{N}}^{-}\right)$has an atom given by:

$$
\left|\dot{U}_{\mathrm{N}}^{+}\right|^{2}-\left|\dot{U}_{\mathrm{N}}^{-}\right|^{2}=\left(e^{2}-1\right)\left|\dot{U}_{\mathrm{N}}^{-}\right|^{2} \leq 0
$$

thanks to the equation of motion and the impact law. 
Taking these two remarks into account in (9), we obtain:

$$
\int_{[0, t]}\left(R_{\mathrm{N}}-R_{\mathrm{aN}}\right)\left(\left(\dot{U}_{\mathrm{N}}^{+}+\dot{U}_{\mathrm{N}}^{-}\right) / 2-\dot{U}_{\mathrm{aN}}\right) \leq-\int_{0}^{t} R_{\mathrm{aN}}\left(\dot{U}_{\mathrm{N}}^{+}-\dot{U}_{\mathrm{aN}}\right)
$$

and then:

$$
\frac{1}{2}\left|\dot{U}_{\mathrm{N}}^{+}-\dot{U}_{\mathrm{aN}}\right|^{2}(t)+\frac{k_{\mathrm{N}}}{2}\left|U_{\mathrm{N}}-U_{\mathrm{aN}}\right|^{2}(t) \leq|W| \int_{0}^{t}\left|U_{\mathrm{T}}-U_{\mathrm{aT}}\right|\left|\dot{U}_{\mathrm{N}}^{+}-\dot{U}_{\mathrm{aN}}\right|+\int_{0}^{t}\left|R_{\mathrm{aN}}\right|\left|\dot{U}_{\mathrm{N}}^{+}-\dot{U}_{\mathrm{aN}}\right| .
$$

Lemma A.5, p. 157 of [2] allows us to obtain the desired estimate:

$$
\left|\dot{U}_{\mathrm{N}}^{+}-\dot{U}_{\mathrm{aN}}\right|(t)+\left|U_{\mathrm{N}}-U_{\mathrm{aN}}\right|(t) \leq \frac{k_{\mathrm{N}}+1}{k_{\mathrm{N}}}|W| \sqrt{2} \int_{0}^{t}\left|U_{\mathrm{T}}-U_{\mathrm{aT}}\right|+\frac{k_{\mathrm{N}}+1}{k_{\mathrm{N}}} \sqrt{2} \int_{0}^{t}\left|R_{\mathrm{aN}}\right| .
$$

Step 3. For all $t \in\left[0, T_{\mathrm{a}}[\right.$, the following estimate holds:

$$
\int_{[0, t]}\left|R_{\mathrm{N}}-R_{\mathrm{aN}}\right| \leq\left|\dot{U}_{\mathrm{N}}^{+}-\dot{U}_{\mathrm{aN}}\right|(t)+C_{5} \int_{0}^{t}\left|U_{\mathrm{N}}-U_{\mathrm{aN}}\right|+C_{6} \int_{0}^{t}\left|U_{\mathrm{T}}-U_{\mathrm{aT}}\right|+2 \int_{0}^{t}\left|R_{\mathrm{aN}}\right|,
$$

for some real constants $C_{5}$ and $C_{6}$ depending only on $K$.

Since $R_{\mathrm{N}}$ is a nonpositive measure:

$$
\int_{[0, t]}\left|R_{\mathrm{N}}-R_{\mathrm{aN}}\right| \leq-\int_{[0, t]} R_{\mathrm{N}}+\int_{0}^{t}\left|R_{\mathrm{aN}}\right|
$$

Also, we have:

$$
\begin{aligned}
-\int_{[0, t]} R_{\mathrm{N}} & =-\int_{[0, t]}\left(\ddot{U}_{\mathrm{N}}-\ddot{U}_{\mathrm{aN}}\right)-\int_{0}^{t} k_{\mathrm{N}}\left(U_{\mathrm{N}}-U_{\mathrm{aN}}\right)+W \cdot\left(U_{\mathrm{T}}-U_{\mathrm{aT}}\right)+R_{\mathrm{aN}} \\
& \leq\left|\dot{U}_{\mathrm{N}}^{+}-\dot{U}_{\mathrm{aN}}\right|(t)+k_{\mathrm{N}} \int_{0}^{t}\left|U_{\mathrm{N}}-U_{\mathrm{aN}}\right|+|W| \int_{0}^{t}\left|U_{\mathrm{T}}-U_{\mathrm{aT}}\right|+\int_{0}^{t}\left|R_{\mathrm{aN}}\right| .
\end{aligned}
$$

Putting everything together, we get the estimate that we looked for, with $C_{5}=k_{\mathrm{N}}$ and $C_{6}=|W|$.

Step 4. For all $t \in\left[0, T_{\mathrm{a}}[\right.$, the following estimate holds:

$$
\left|\dot{U}_{\mathrm{T}}^{+}-\dot{U}_{\mathrm{aT}}\right|(t)+\left|U_{\mathrm{T}}-U_{\mathrm{aT}}\right|(t) \leq C \int_{0}^{t}\left|R_{\mathrm{aN}}\right|,
$$

for some real constant $C$ depending only on $K, \mu$ and $T_{\mathrm{a}}$.

Putting together steps 1, 2 and 3, the function:

$$
\phi(t) \stackrel{\text { def }}{=}\left|\dot{U}_{\mathrm{T}}^{+}-\dot{U}_{\mathrm{aT}}\right|(t)+\left|U_{\mathrm{T}}-U_{\mathrm{aT}}\right|(t)
$$

satisfies the estimate:

$$
\phi(t) \leq C_{7} \int_{0}^{t} \phi+C_{8} \int_{0}^{t}\left|R_{\mathrm{aN}}\right|,
$$


for some real constants $C_{7}$ et $C_{8}$ depending only on $K, \mu$ and $T_{\mathrm{a}}$. By Gronwall's lemma, we get:

$$
\begin{aligned}
\phi(t) & \leq C_{8} \int_{0}^{t}\left|R_{\mathrm{aN}}\right|+C_{7} C_{8} \int_{0}^{t} e^{C_{7}(t-s)} \int_{0}^{s}\left|R_{\mathrm{aN}}\right|, \\
& \leq C_{8}\left(1+C_{7} T_{\mathrm{a}} e^{C_{7} T_{\mathrm{a}}}\right) \int_{0}^{t}\left|R_{\mathrm{aN}}\right|
\end{aligned}
$$

which is the estimate that we sought.

Step 5. Conclusion.

The function $R_{\mathrm{aN}}$ being analytic, only the two following cases are possible.

(1) $R_{\mathrm{aN}} \equiv 0$. In such a case, step 4 gives $U_{\mathrm{T}} \equiv U_{\mathrm{aT}}$ and step $2, U_{\mathrm{N}} \equiv U_{\mathrm{aN}}$. The sought conclusion holds true.

(2) $\left.\forall t \in] 0, T_{a}\right], \quad R_{\mathrm{aN}}(t)<0$. Then, the function $U_{\mathrm{aN}}$ vanishes identically. Since the uniqueness of solution has already been proved for the bilateral problem, it is enough to prove $U_{\mathrm{N}} \equiv 0$ to reach the desired conclusion. So, let us concentrate on this goal. Taking $T_{\mathrm{a}}$ smaller, if necessary, we have:

$$
\left.\forall t \in] 0, T_{\mathrm{a}}\right], \quad-R_{\mathrm{aN}}(t)-C|W| \int_{0}^{t}\left|R_{\mathrm{aN}}\right|>0 .
$$

Multiplying the equation:

$$
\ddot{U}_{\mathrm{N}}+k_{\mathrm{N}} U_{\mathrm{N}}=R_{\mathrm{N}}-R_{\mathrm{aN}}-W \cdot\left(U_{\mathrm{T}}-U_{\mathrm{aT}}\right),
$$

by $\left(\dot{U}_{\mathrm{N}}^{+}+\dot{U}_{\mathrm{N}}^{-}\right) / 2$ and integrating over $[0, t]$, we obtain:

$$
\frac{1}{2}\left|\dot{U}_{\mathrm{N}}^{+}\right|^{2}(t)+\frac{k_{\mathrm{N}}}{2}\left|U_{\mathrm{N}}\right|^{2}(t)=\int_{[0, t]} R_{\mathrm{N}} \frac{\dot{U}_{\mathrm{N}}^{+}+\dot{U}_{\mathrm{N}}^{-}}{2}-\int_{0}^{t}\left(R_{\mathrm{aN}}+W \cdot\left(U_{\mathrm{T}}-U_{\mathrm{aT}}\right)\right) \dot{U}_{\mathrm{N}}^{+} .
$$

Since $R_{\mathrm{N}}\left(\dot{U}_{\mathrm{N}}^{+}+\dot{U}_{\mathrm{N}}^{-}\right)$is a nonpositive measure, we have:

$$
\int_{0}^{t}\left(R_{\mathrm{aN}}+W \cdot\left(U_{\mathrm{T}}-U_{\mathrm{aT}}\right)\right) \dot{U}_{\mathrm{N}}^{+} \leq 0 .
$$

Applying an integration by parts, we get:

$$
\left(R_{\mathrm{aN}}+W \cdot\left(U_{\mathrm{T}}-U_{\mathrm{aT}}\right)\right) U_{\mathrm{N}} \leq \int_{0}^{t}\left(\dot{R}_{\mathrm{aN}}+W \cdot\left(\dot{U}_{\mathrm{T}}^{+}-\dot{U}_{\mathrm{aT}}\right)\right) U_{\mathrm{N}},
$$

and, therefore, by step 4:

$$
0 \leq\left\{\left|R_{\mathrm{aN}}\right|(t)-C|W| \int_{0}^{t}\left|R_{\mathrm{aN}}\right|\right\}\left|U_{\mathrm{N}}\right|(t) \leq \int_{0}^{t}\left\{\left|\dot{R}_{\mathrm{aN}}\right|(s)+C|W| \int_{0}^{s}\left|R_{\mathrm{aN}}\right|\right\}\left|U_{\mathrm{N}}\right|(s) .
$$

Denoting by $m \in \mathbb{N}$ the order of the first nonzero term in the power series expansion at 0 of the analytic function $R_{\mathrm{aN}}$, we get the estimate:

$$
\forall t \in] 0, T_{\mathrm{a}}\left[, \quad\left|\dot{R}_{\mathrm{aN}}\right|(t) \leq \frac{m+\tilde{D} t}{t}\left|R_{\mathrm{aN}}\right|(t),\right.
$$


for some nonnegative real constant $\tilde{D}$. We deduce that the following estimate holds:

$$
\forall t \in] 0, T_{\mathrm{a}}\left[, \quad\left|\dot{R}_{\mathrm{aN}}(t)+C\right| W\left|\int_{0}^{t}\right| R_{\mathrm{aN}}|| \leq \frac{m+D t}{t}|| R_{\mathrm{aN}}|(t)-C| W\left|\int_{0}^{t}\right| R_{\mathrm{aN}}||,\right.
$$

for some nonnegative real constant $D$. Substituting this estimate into the previous inequality, we get:

$$
t \psi(t) \leq(m+D t) \int_{0}^{t} \psi
$$

where we have set:

$$
\psi(t) \stackrel{\text { def }}{=}\left\{\left|R_{\mathrm{aN}}\right|(t)-C|W| \int_{0}^{t}\left|R_{\mathrm{aN}}\right|\right\} \frac{\left|U_{\mathrm{N}}\right|(t)}{t},
$$

which is a continuous function, even at $t=0$ (more precisely $\psi(t)=o\left(t^{m}\right)$ when $t \rightarrow 0$ ). Then, we see that:

$$
\forall t \in] 0, T_{\mathrm{a}}\left[, \quad \frac{\mathrm{d}}{\mathrm{d} t}\left\{\frac{e^{-D t}}{t^{m}} \int_{0}^{t} \psi\right\} \leq 0,\right.
$$

which implies that this nonnegative function which vanishes at $t=0$ is nonincreasing. Therefore:

$$
\psi \equiv 0,
$$

and, then:

$$
U_{\mathrm{N}} \equiv 0
$$

which is nothing but the desired conclusion.

\subsection{Well-posedness of the unilateral problem with analytic force}

Corollary 4.3. If $F:[0, T] \rightarrow \mathbb{R}^{n}$ is analytic (or piecewise analytic), then, problem $\mathcal{P}_{\mathrm{u}}$ admits a unique solution in $\operatorname{MMA}\left([0, T] ; \mathbb{R}^{n}\right)$.

Proof. Using the local existence of solution for problem $\mathcal{P}_{\mathrm{u}}$ provided by Theorem 4.1, and the local uniqueness in $M M A$, provided by Theorem 4.2, we get a maximal solution $U$ which is defined either on a subinterval [0, $\eta[$, for some $\eta \in] 0, T]$, or on $[0, T]$. We have to prove that it is defined on $[0, T]$. To reach this conclusion, it is enough to prove that, if the maximal solution were defined only on a subinterval $[0, \eta[$, then, the total variation of the right-velocity $\dot{U}^{+}$over $[0, \eta[$ is finite, for, in such a case, it would be possible to extend $U$ beyond $[0, \eta[$ and obtain a contradiction. So, suppose that the maximal solution is only defined on $[0, \eta[$.

First, we have already noticed that $R_{\mathrm{N}}\left(\dot{U}_{\mathrm{N}}^{+}+\dot{U}_{\mathrm{N}}^{-}\right)$is a nonpositive measure, thanks to the contact condition, the equation of motion and the impact law. Also, by the Coulomb friction law, $R_{\mathrm{T}} \cdot \dot{U}_{\mathrm{T}}^{+}$is a nonpositive measure. Let $D$ be the countable subset of those instants $t \in[0, \eta[$ at which the tangential velocity is discontinuous: $\dot{U}_{\mathrm{T}}^{+}(t) \neq \dot{U}_{\mathrm{T}}^{-}(t)$. On $\left[0, \eta\left[\backslash D\right.\right.$, the measure $R_{\mathrm{T}} \cdot\left(\dot{U}_{\mathrm{T}}^{+}+\dot{U}_{\mathrm{T}}^{-}\right)$equals $R_{\mathrm{T}} \cdot \dot{U}_{\mathrm{T}}^{+}$which is nonpositive. At each instant $t \in D$, making use of the equation of motion together with the Coulomb friction law, it is readily checked that the measure $R_{\mathrm{T}} \cdot\left(\dot{U}_{\mathrm{T}}^{+}+\dot{U}_{\mathrm{T}}^{-}\right)$has a negative atom. Finally, $R \cdot\left(\dot{U}^{+}+\dot{U}^{-}\right)$is a nonnegative measure. So, multiplying the equation of motion:

$$
\ddot{U}+K \cdot U=F+R
$$

by $\left(\dot{U}^{+}+\dot{U}^{-}\right) / 2$, and integrating over $\left.] 0, t\right](t \in] 0, \eta[)$, we get the energy inequality:

$$
\frac{1}{2}\left|\dot{U}^{+}\right|^{2}(t)+\frac{1}{2}|U|_{K}^{2}(t) \leq \frac{1}{2}\left|V_{0}\right|^{2}+\frac{1}{2}\left|U_{0}\right|_{K}^{2}+\int_{0}^{t} F \cdot \dot{U}^{+}
$$


Applying Lemma A.5, p. 157 of [2]:

$$
\left|\dot{U}^{+}\right|(t) \leq\left|U_{0}\right|_{K}+\left|V_{0}\right|+\int_{0}^{T}|F|
$$

we find that the right-velocity $\dot{U}^{+}$is bounded over the interval $[0, \eta[$. Next, integrating the first component of the equation of motion over $[0, t](t \in] 0, \eta[)$, we get:

$$
\int_{[0, t]} R_{N}=\dot{U}_{\mathrm{N}}^{+}(t)-V_{0 \mathrm{~N}}+\int_{0}^{t}\left(k_{\mathrm{N}} U_{\mathrm{N}}+W \cdot U_{\mathrm{T}}\right)-\int_{0}^{t} F_{\mathrm{N}},
$$

which, since the right-velocity $\dot{U}^{+}$is bounded over [0, $\eta$ and since $R_{N}$ is a nonpositive measure, shows:

$$
\int_{[0, \eta[}\left|R_{\mathrm{N}}\right|<\infty
$$

But, Coulomb friction law implies:

and therefore:

$$
\left|R_{\mathrm{T}}\right| \leq \mu\left|R_{\mathrm{N}}\right|
$$

$$
\int_{[0, \eta[}|R|<\infty .
$$

Coming back to the equation of motion, we obtain:

$$
\int_{[0, \eta[}|\ddot{U}|<\infty
$$

which is the desired conclusion.

Acknowledgements. We are thankful to Alain Léger for valuable discussions and constant support during this work.

\section{REFERENCES}

[1] P. Ballard, The dynamics of discrete mechanical systems with perfect unilateral constraints. Arch. Rational Mech. Anal. 154 (2000) 199-274.

[2] H. Brezis, Opérateurs Maximaux Monotones et Semi-groupes de Contractions dans les Espaces de Hilbert. North-Holland Publishing Company (1973).

[3] H. Cartan, Théorie Élémentaire des Fonctions Analytiques d'une ou plusieurs Variables Complexes. Hermann, Paris (1961).

[4] A. Klarbring, Ingenieur-Archiv 60 (1990) 529-541.

[5] M.D.P. Monteiro Marques, Differential Inclusions in Nonsmooth Mechanical Problems. Birkhaüser, Basel-Boston-Berlin (1993).

[6] J.J. Moreau, Standard inelastic shocks and the dynamics of unilateral constraints, in Unilateral problems in structural analysis. G. Del Piero and F. Maceri Eds., Springer-Verlag, Wien-New-York (1983) 173-221.

[7] J.J. Moreau, Dynamique de systèmes liaisons unilatérales avec frottement sec éventuel : essais numériques. Note Technique No 85-1 (1985), LMGMC, Montpellier.

[8] J.J. Moreau, Unilateral contact and dry friction in finite freedom dynamics, in Nonsmooth Mechanics and Applications, CISM Courses and Lectures No 302, J.J. Moreau and P.D. Panagiotopoulos Eds., Springer-Verlag, Wien-New-York (1988) 1-82.

[9] J.J. Moreau, Bounded variation in time, in Topics in Non-smooth Mechanics, J.J. Moreau, P.D. Panagiotopoulos, G. Strang, Eds., Birkhaüser Verlag, Basel-Boston-Berlin (1988) 1-74.

[10] D. Percivale, Uniqueness in the elastic bounce problem, I. J. Differ. Equations 56 (1985) 206-215.

[11] M. Schatzman, A class of nonlinear differential equations of second order in time. Nonlinear Anal. Theory, Methods Appl. 2 (1978) 355-373.

[12] M. Schatzman, Uniqueness and continuous dependence on data for one dimensional impact problems. Math. Comput. Modelling 28 (1998) $1-18$. 